Journal of the Operations Research Society of Japan

Vol. 28, No. 4, December 1985

\title{
A PRODUCTION PLANNING MODEL FOR MULTI-PRODUCT FACILITIES
}

\author{
Chang Sup Sung \\ Korea Advanced Institute of Science \& Technology
}

(Received April 5, 1985: Final September 18, 1985)

\begin{abstract}
A production planning model for multi-product facilities is analyzed, in which known demands must be satisfied. In the model, in every production period each facility produces a certain number of items each taking a fixed part of the production amount. Concave production costs dependent upon the production in different facilities and piecewise concave inventory costs are considered. Both the nonbacklog and backlog permitted cases are considered. The structure of an optimal solution is characterized and then used illustratively in a simple dynamic programming algorithm for nonbacklog single-facility problems.
\end{abstract}

\section{Introduction}

Wager and Whitin [2] have analyzed an important single-product, singlefacility production and inventory problem. Assuming that demands for the product were known, they sought to find a production schedule, in terms of how much to produce in each period for the next $N$ periods, that minimized the cost of producing and holding inventory. They further assumed that the production and inventory cost functions were concave and that no backlogging of unsatisfied demand was permitted.

Florian and Klein [1] have considered the problem of Wagner and Whitin [2] under the assumption that the production levels are restricted to the perioddependent capacity limits. They have also considered the backlogging case in the work, while a dynamic programming algorithm was suggested only for the problems with a fixed capacity limit.

Zangwill [3] has considered a multi-product, multi-facility model, which is, however, a linking together of the single-product single-facility model. He has also studied a multi-echelon model only for a single product in another paper [4]. 
In this paper, three different cases of production planning for multiproduct facilities will be analyzed. The first two cases are for the problem of multi-product single-facility production planning with and without backlog, respectively, and the last one is for multi-product multi-facility production planning problems with backlog that is essentially a linking together of the backlogged single-facility cases and so forms an acyclic network of the facilities. In each case, in every production period each facility produces multiple items each taking a fixed part of the whole production amount. As an example, an oil refinery problem can be taken into account, where some amount of crude oil may be refined to produce two different items, gasoline and some fine chemical resources, in the fixed production amount ratios, say $\alpha_{1}>0$ and $\alpha_{2}>0$ percentages, respectively. Concave production costs dependent upon the production in different facilities and piecewise concave inventory costs are considered.

The objective of this paper is to find a useful description of the structure of optimal plans for each case. They consist of independent subplans in which in each production period except the last, where all the demands are exactly satisfied, at least one of the demands for different items is exactly satisfied. Each feasible plan consisting only of such subplans is proven to be an extreme point of the feasible production schedules set for the singlefacility problems and also to be an element of the dominant set (defined by Zangwill [3]) for the multi-facility problem. This characterization is then illustrated by a simple dynamic programming algorithm for the nonbacklogged single-facility problem.

\section{Single-Facility Case with Nonbacklog}

\subsection{Model formulation}

Consider a $M$-product problem with each product $i$ (item $i)(i=1,2, \ldots, M)$ taking $\alpha_{i}>0$ parts of the total production amount in every period. That is, the amount produced in each period $\left(x_{1}, x_{2}, \ldots, x_{N}\right)=x$ has the relations

$$
x_{t}=\sum_{i=1}^{M} x_{t i} \text { and } x_{t i}=x_{t}=\left(\alpha_{i} / M_{\alpha}\right)
$$

where $M_{\alpha}=\sum_{j=1}^{M} \alpha_{j}$, and $x_{t i}$ is the production amount of item $i$ in period $t(t=1,2, \ldots, N ; i=1,2, \ldots, M)$. Let $r_{1}, \ldots, r_{N}$ represent the known demands over the planning horizon $N$, where $r_{t}(t=1, \ldots, N)$ is the demand vector in period $t$, 
$r_{t}=\left(r_{t 1}, \ldots, r_{t M}\right)$. The component $r_{t i} \geq 0$ represents the demand for item $i$ in period $t$. Note that each demand $r_{t i}$ is not necessarily required to take $\alpha_{i}$ parts of the total demand in period $t$.

There is no loss in generality in assuming that both the initial inventory and the final inventory are zeros. Its reason is that if the equality of " $R_{1 N}(i) / \sum_{j=1}^{M} R_{1 N}(j)=\alpha_{i} / M_{\alpha} "$ (where $R_{\ell m}(i)=\sum_{t \equiv \ell}^{m} r_{t i}$ ) does not hold for any $i$, some additional (artificial) demands can be considered to adjust each of the last demands to the equality. Then, the problem is to minimize the total costs of production and inventory, say

$$
G(X)=\sum_{t=1}^{N} C_{t}\left(x_{t}\right)+\sum_{t=1}^{N} \sum_{i=1}^{M} H_{t i}\left(I_{t i}\right)
$$

subject to

$$
\begin{aligned}
& I_{t i}=x_{1 t}(i)-R_{1 t}(i), \forall i \text { and } t \\
& x_{t}=\sum_{i=1}^{M} x_{t i}, \quad \forall t
\end{aligned}
$$

$$
\begin{aligned}
& x_{t i}=\left(\alpha_{i} / \alpha_{1}\right) \quad x_{t 1}, \quad \forall i \text { and } t \\
& I_{t i} \geq 0 \text { and } x_{t i} \geq 0, \quad \forall i \text { and } t \\
& I_{0 i}=0=I_{N i}, \forall i,
\end{aligned}
$$

where $X_{\ell m}(i)=\sum_{t=\ell}^{m} x_{t i},(\ell=1, \ldots, N-1 ; m=\ell, \ell+1, \ldots, N)$, and $c_{t}$ is the concave production cost function for period $t$, and $H_{t i}$ gives the concave cost of storing the inventory quantity $I_{t i}$ of item $i$ from period $t$ to period $t+1$.

The constraints of the problem $\left(\mathrm{P}_{1}\right)$ can be depicted for period $t$ as in Fig. 1, where $I_{t}$ is the inventory vector in period $t, I_{t}=\left(I_{t 1}, \ldots, I_{t M}\right)$.

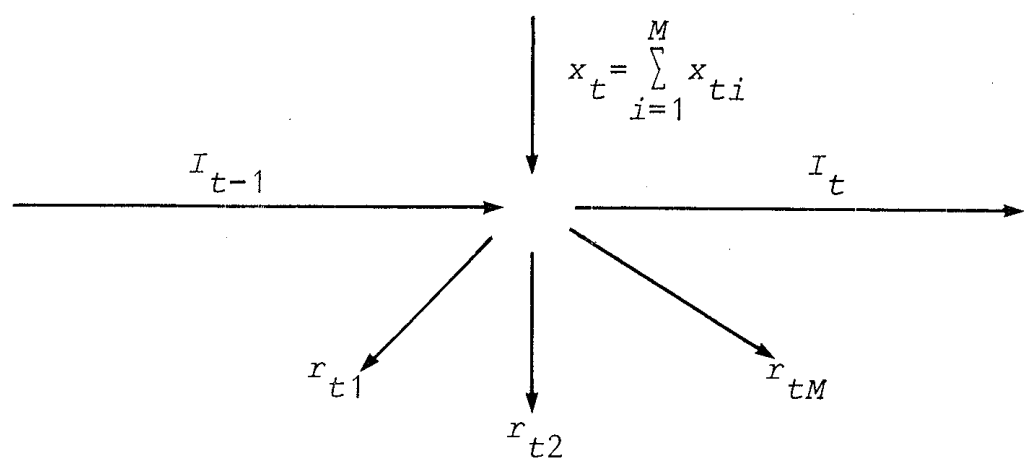

Fig. 1. A Network for a M-Product Single-Facility Mode1 
The constraints of the problem $\left(P_{1}\right)$ define a closed bounded convex set. Since $G$ is concave, we know that it attains its minimum at an extreme point of the set. Let $D$ be the set of all the extreme points of the feasible solutions set. The characterization of $D$ will then be made in the next section, which will be used to facilitate finding an optimal production plan.

Consider the following inventory decomposition property (see Florian and Klein [1]) for its proof) upon which our approach is based.

Theorem 1. (Inventory Decomposition Property). Suppose that the constraints, $I_{\ell, i}=0$ for all $i$ and some $\ell \varepsilon\left\{1,2, \ldots, N^{-1}\right\}$, are added to those described in the problem $\left(\mathrm{P}_{1}\right)$. Then, an optimal solution to the original problem can be found by independently finding solutions to the problems for the first $\ell$ periods and for the last $N$-l periods.

\subsection{Characterization of extreme point}

Let us introduce an "at-least-one exact requirement sequence" that wil1 form the basis for our characterization of $D$.

Definition. A production sequence $\left(x_{1}, x_{2}, \ldots, x_{N}\right)$ is called an "at-leastone exact requirement sequence" if for every $n(1 \leq n<N)$ such that $x_{n+1}>0$, $\sum_{t=1}^{n} x_{t}=L(n)$, where $L(n)=\max _{i}\left\{\left(M_{\alpha} / \alpha_{i}\right) R_{1 n}(i)\right\}$.

Note that $L(n)$ is equal to the lower bound of cumulative production amount from period 1 to period $n$. Each feasible plan forming an at-least-one exact requirement sequence is then characterized as the correspondence to an element of $D$.

Theorem 2. A feasible plan $x$ is an "at-least-one exact requirement sequence" iff it is in $D$.

Proof: Suppose $X \in D$, and $X$ does not form an at-least-one exact require-ment sequence. Hence, there is at least one production period, say $b, 1 \leq b<N$, in which for every $i \varepsilon\{1,2, \ldots, M\}$,

$$
R_{1, b-1}(i)<X_{1, \sigma(b)}(i)
$$

and further, among them at least one item $j$ is in "partial" inventory, i.e., $0<I_{b-1, j}<r_{b j}$, where $\sigma(b)$ is the last production period prior to period $b$. Without loss of generality, we suppose that there is just one such period.

Let

$$
\delta=\frac{1}{2} \min \left[\min _{j \in J}\left[\left(\alpha_{1} / \alpha_{j}\right) I_{b-1, j},\left(\alpha_{1} / \alpha_{j}\right)\left(r_{b j}-I_{b-1, j}\right)\right], \min _{j \in J}\left[\left(\alpha_{1} / \alpha_{j}\right) I_{b-1, j}\right]\right]
$$


where $J$ is the set of partial inventory items, $\vec{J}$ is the complement of $J$ and $\left(\alpha_{1} / \alpha_{j}\right) I_{b-1, j}$ is the inventory amount of item $j$ computed in terms of item 1 , and let $U_{t}$ be a $N$ component vector with a unity element in the $t^{\text {th }}$ position, and zeros elseqhere.

Now, define the distinct production plans

$$
\begin{aligned}
& x^{\prime}=x-\delta U_{\sigma(b)}+\delta U_{b} \text { and } \\
& x^{\prime \prime}=x+\delta U_{\sigma(b)}-\delta U_{b} .
\end{aligned}
$$

Since $\delta>0$, these plans are easily seen to be feasible. However, $x=1 / 2$ $\left(x^{\prime}+x^{\prime \prime}\right)$, contradicting our assumption that $x$ is an extreme point. This proves "if part".

Suppose on the contrary that $x \notin D$. Then, there are feasible distinct plans $x^{\prime}$ and $x^{\prime \prime}$ such that $x=\left(x^{\prime}+x^{\prime \prime}\right)$. This implies, however, that by hypothesis there exists an item $j \varepsilon\{1,2, \ldots, M\}$ such that in a production period $b(1 \leq b \leq N), \quad\left(\alpha_{j} / M_{\alpha}\right) L(b-1)=R_{1, b-1}(j)$ and so either $I_{b-1, j}^{\prime}<0$ or $I_{b-1, j}^{\prime \prime}<0$, where $I_{b-1, j}^{\prime}$ and $I_{b-1, j}^{\prime \prime}$ represent the inventory levels of item $j$, at the end of period $b-1$, associated with the two plans $x^{\prime}$ and $x^{\prime \prime}$, respectively. This contradicts the assumption that both $X^{\prime}$ and $X^{\prime \prime}$ are feasible.

Thus, the proof is completed.

Theorem 2 implies that the set of all at-least-one exact requirement sequences is $D$. Therefore, one of such sequences having the minimum cost becomes and optimal solution.

\subsection{An Algorithm}

Before developing our algorithm it is necessary to specify how to determine each component $x_{t}{ }^{*}(t=0,1, \ldots, N)$ of an optimal solution $X^{*}$ in somewhat more detail.

The descriptions of the elements of $D$ given in Theorem 2 can be used to facilitate finding an optimal plan $X^{*}$. It follows from Theorem 2 that all extreme point solutions, including the optimal one, have the property that $M$ $\prod_{i=1} I_{t-1, i^{X}}=0(t=1,2, \ldots, N)$. This leads to Corollary 1.

Corollary 1. An optimal production plan $x *$ consists of component $x_{t}^{* \prime}$ s such that $\prod_{i=1} I_{t-1}, i_{t}^{* *=0}$ for $t=1,2, \ldots, N$.

Denote by $x_{m+1(n)}$ the amount of the production completed in period $m+1$, $0 \leq m<n \leq N$, to satisfy the demands over the periods from $m^{+1}$ through $n$, where for 
al1 $i, I_{t i}>0(m<t<N), \prod_{i=1}^{M} I_{m i}=0$ and $\prod_{i=1}^{M} I_{n i}=0$. Then, each optimal quantity $x_{m+1(n)}^{*}$ can be determined immediately based on the results of Theorem 1 and Corollary 1 .

Theorem 3. An optimal production plan $x^{*}$ consists only of component $x_{m}^{* s} s(m=0,1, \ldots, N-1)$ satisfying the relation

$$
x_{m+1(n)}^{*}=L(n)-L(m) \text { for } 0 \leq m<n \leq N
$$

The results of Theorem 3 indicate that an optimally production plan can be found by searching optimality for $(m, n)$ sequences. Thereupon, a dynamic programming recursion shall be exploited.

Let $d_{m n}$ denote the production and inventory costs associated with producing the amount of $x_{m+1(n)}$. Then, $d_{m n}$ can be expressed as follows:

$$
a_{m n}=c_{m+1}\left(x_{m+1(n)}\right)+\sum_{t=m+1}^{n} \sum_{j=1}^{M}{ }^{H}{ }_{t j}\left(I_{t j}\right),
$$

where $I_{t j}(m+1 \leq t \leq n)$ are the inventory levels associated with production $x_{m+1(n)}$ in period $m+1$.

The results of Theorem 3 and $d_{m n}$ values can be applied to form a dynamic programming recursion using the periods $0,1,2, \ldots, N$ as states. Let $F_{t}$ be the cost associated with an optimal production plan over periods $0,1,2, \ldots, t$ $(t=0,1, \ldots, N)$, given that $\prod_{i=1}^{M} I_{t i}=0$.
Then,

Then,

$$
F_{n}=\min _{0 \leqq m \leq n-1}\left[F_{m}+d_{m n}\right], \quad(n=1,2, \ldots, N)
$$

and $F_{0} \equiv 0$.

The recursion (3) indicates that the minimum cost for the first $n$ periods comprises the setup and production costs in period $m+1$, the charges for filling demand $r_{t}\left(t=m^{+1}, \ldots, n\right)$ by carrying inventory from period $m^{+1}$, and the cost of adapting an optimal policy in periods 0 through $m$ taken by themselves. Theorems 2 and 3 guarantee that at period $n$ we shall find an optimum plan (schedule) of this type.

Now, the results of Theorem 3 and the relations (2) and (3) are put together to give the following step-by-step description of the solution procedure:

Step 1 (Initialization): Set $F_{0}=0, n=1$, and $m=0$.

Step 2: Compute $a_{m n}$ values for each $m(0 \leq m \leq n-1)$ by use of Theorem 3 and the relation (2), and repeat Step 2 with " $m=m+1$ " until " $m=n-1$ ". 
Step 3: Apply the recursion (3) for finding each optimal policy $F_{n}$, and repeat Steps 2 and 3 with " $n=n+1$ " until the last period " $n=N$ ".

The algorithm described above indicates that to determine an optimal policy $F_{n}$ in period $n$ by use of the recursion ( 3$)$, in general, $n(n+1) / 2$ computations for the values of $d_{m n}$ 's $(0 \leq m \leq N-1)$ are required. Therefore, finding an optimal plan $X^{*}$ is, in general, a tedious combinatorial problem when $N$ is large.

\subsection{An example}

We illustrate the algorithm with the 6 period two-product single-facility problem and with the production ratio of ${ }^{\alpha_{1}}: \alpha_{2}=2: 3$ ". The production and inventory cost functions are given as follows:

$$
\begin{aligned}
& C_{t}\left(x_{t}\right)=5(7-t) \delta\left(x_{t}\right)+10 x_{t}, \\
& H_{t 1}\left(I_{t 1}\right)=3 t I_{t 1}, \text { and } \\
& H_{t 2}\left(I_{t 2}\right)=2 t I_{t 2},
\end{aligned}
$$

where $\delta\left(x_{t}\right)=\left\{\begin{array}{ll}0, & \text { if } x_{t}=0 \\ 1, & \text { if } x_{t}>0\end{array}, t=1,2, \ldots, 6\right.$.

The demands for $\left\{r_{t 1}\right\}$ are $(3,2,5,4,3,7)$, and for $\left\{r_{t 2}\right\}$ are $(5,6,4,7,6,8)$. Then, these demands are tabulated in Table 1 to figure out values of $L(n)$ and $L(n)-L(m)$ for $x_{m+1(n)}^{*}$ computation at each $m(0 \leq m<n \leq N)$. The last row of Table

\begin{tabular}{|c|c|c|c|c|c|c|c|c|c|c|c|}
\hline \multirow{3}{*}{$n$} & \multirow{3}{*}{$r_{n 1}$} & \multirow{3}{*}{$r_{n 2}$} & \multirow{3}{*}{$\frac{{ }^{M}}{\alpha_{1}^{\alpha}} R_{1 n}(1)$} & \multirow{3}{*}{$\frac{M_{\alpha}}{\alpha_{2}} R{ }_{1 n}(2)$} & \multirow{3}{*}{$L(n)$} & \multicolumn{6}{|c|}{$L(n)-L(m)$} \\
\hline & & & & & & \multicolumn{6}{|c|}{$m$} \\
\hline & & & & & & 0 & 1 & 2. & 3 & 4 & 5 \\
\hline 1 & 3 & 5 & $15 / 2$ & $25 / 3$ & $25 / 3$ & $25 / 3$ & & & & & \\
\hline 2 & 2 & 6 & $25 / 2$ & $55 / 3$ & $55 / 3$ & $55 / 3$ & 10 & & & & \\
\hline 3 & 5 & 4 & 25 & 25 & 25 & 25 & $50 / 3$ & $20 / 3$ & & & \\
\hline 4 & 4 & 7 & 35 & $110 / 3$ & $110 / 3$ & $110 / 3$ & $85 / 3$ & $55 / 3$ & $35 / 3$ & & \\
\hline 5 & 3 & 6 & $85 / 2$ & $140 / 3$ & $140 / 3$ & $140 / 3$ & $115 / 3$ & $85 / 3$ & $65 / 3$ & 10 & \\
\hline 6 & 7 & 8 & 60 & 60 & 60 & 60 & $155 / 3$ & $125 / 3$ & 35 & $70 / 3$ & $40 / 3$ \\
\hline \multicolumn{6}{|c|}{$x_{m+1}^{*}(n)$} & $\begin{array}{c}x_{1}^{*}= \\
55 / 3\end{array}$ & $\begin{array}{r}x_{2}^{*}= \\
0\end{array}$ & $\begin{array}{c}x_{3}^{*}= \\
20 / 3\end{array}$ & $\begin{array}{c}x_{4}^{*}= \\
35 / 3\end{array}$ & $\begin{array}{l}x_{5}^{*}= \\
10\end{array}$ & $\begin{array}{c}x * \frac{*}{6}= \\
40 / 3\end{array}$ \\
\hline
\end{tabular}
1 shows the optimal solution $X^{*}$.

Table 1. Demand Data 
The solution procedure in section 2.3 is then applied to the example, and $F_{1}, F_{2}, F_{3}, F_{4}, F_{5}$ and $F_{6}$ are calculated as follows: $F_{1}=F_{0}+d_{01}=0+343 / 3=343 / 3$ with $x_{1(1)}^{*}=25 / 3 ; F_{2}=\min \left\{F_{0}+d_{02}, F_{1}+d_{12}\right\}=F_{0}+d_{02}=757 / 3$ with $x_{1(2)}^{*}=55 / 3 ; F_{3}=$ $\min _{0 \leqq m \leqq 2}\left\{F_{m}+d_{m 3}\right\}=F_{2}+d_{23}=1017 / 3$ with $x_{3(3)}^{*}=20 / 3 ; F_{4}=\min _{0 \leqq m \leqq 3}\left\{F_{m}+d_{m 4}\right\}=F_{3}+d_{34}=1436 / 3$

with $x_{4(4)}^{*}=35 / 3 ; F_{5}=\min _{0 \leqq m \leqq 4}\left\{F_{m}+d_{m 5}\right\}=F_{4}+d_{45}=1841 / 3$ with $x_{5(5)}^{*}=10 ; F_{6}=\min _{0 \leqq m \leqq 5}\left\{F_{m}+d_{m 6}\right\}$ $=F_{5}+d_{56}=2256 / 3$ with $x_{6(6)}^{*}=40 / 3$. Thus, the optimal solution of the problem is $x^{*}=(55 / 3,0,20 / 3,35 / 3,10,40 / 3)$.

\section{Single-Facility Case with Backlog}

The model described in section 1 may be extended so that backlogging is permitted. We shall assume as in Zangwill [4] that all demands must be satisfied no longer than $\beta_{j}(j=1,2, \ldots, M)$ periods after the specified delivery data for each item $j$. Thus the constraints $I_{t j} \geq 0$ in the problem (P1) is replaced by

$$
I_{t j} \geq-\sum_{h=t-\beta_{j}+1}^{t} r_{h j}, \text { for a11 } j \text { and } t
$$

$t=\beta_{j}, \beta_{j}+1, \ldots, N$. With backlogging, we assume that for each item $j$ a penalty cost is charged on the amount backlogged in any period and the cost is concave, so that the total inventory cost is piecewise concave.

Although the objective function of ( $\mathrm{P} 1$ ) is then piecewise concave rather than concave, all of the results obtained for the nonbacklog case hold. Since the arguments are, with slight modifications, the same as for the nonbacklog problem, we shall not repeat them in detail. However, we shall indicate noteworthy changes in this section.

The property of Theorem 1 still holds in the case where the backlogging constraints are additionally considered in the problem (P1), so that the recursion (3) may still be used.

Theorem 4. Suppose that the constraints, $I_{\ell j}=0$ for all $i$ and some $\ell \varepsilon\{1,2, \ldots, N-1\}$, are added to those described in (P1) and if $I_{t j} \geq-\sum_{h=t-\beta_{j}+1}^{t} r_{h j}$, $t=\ell+1, \ell+2, \ldots, N$, where $r_{h j}=0$ for $h \leq 0$, then an optimal solution to the extended problem can be found by independently solving the problems for the first $\ell$ periods and for the last $(N-\ell)$ periods.

Following Zangwill [4], the set of all feasible solutions can be partitioned into disjoint subsets, called "basic sets", which are characterized by whether the quantity $I_{t j}$ is nonpositive or positive for each item $j$ in each 
period $t, t=1,2, \ldots, N-1$. Letting $\Omega$ be the set of a11 feasible solutions that satisfy the constraints of the extended problem, then $\Omega$ is the union of a11 $2^{M(N-1)}$ basic sets. Each such subset is closed, bounded and convex with a finite number of extreme points and further, the objective function, $G(X)$, is concave on each given basic set. Thus for a fixed basic set, $G(X)$ attains its minimum at one of its extreme points. Thereupon, letting $D$ be the union of all extreme points of all basic sets, then the minimum of $G(X)$ occurs at a point in $D$.

The above properties lead to our conclusion that the characterization of $D$ for the extended problem can be given similiarly as in Theorem 2 with the newly defined "at-least-one boundary sequence".

Definition. A production sequence $\left(x_{1}, x_{2}, \ldots, x_{N}\right)$ is called an "at-leastone boundary sequence" if for every $n(1 \leq n<N)$ such that $x_{n+1}>0$, $\sum_{t=1}^{n} x_{t} \varepsilon\left\{L(n), L_{\beta}(n)\right\}$, where $L_{\beta}(n)=\max _{i}\left\{\left(M_{\alpha} / \alpha_{i}\right) R_{1}, n-\beta_{i}(i)\right\}$.

Then, each feasible plan forming an at-least-one boundary sequence is characterized as the correspondence to an element of $D$ for the extended problem. This sha11 now be proved.

Theorem 5. A feasible plan $x$ is an "at-least-one boundary sequence" iff it is in $D$.

Proof: The proof of "it part" can be completed easily by following the proof steps of Theorem 2 with the newly defined $\delta^{\prime}$;

$$
\begin{aligned}
\delta^{\prime}= & \frac{1}{2} \min \left[\min _{j \varepsilon J^{+}}\left\{\left(\alpha_{1} / \alpha_{j}\right) I_{b-1, j^{\prime}}\right\}, \min _{j \varepsilon J^{-}}\left\{-\left(\alpha_{1} / \alpha_{j}\right) I_{b-1, j},\right.\right. \\
& \left.\left.\left(\alpha_{1} / \alpha_{j}\right)\left(I_{b-1, j}+\sum_{\ell=b-\beta_{j}}^{b-1} r_{\ell j}\right)\right\}\right],
\end{aligned}
$$

where $J^{+}$and $J^{-}$represent the set of items having on-hand and backlogged inventories, respectively.

For "only if part", suppose on the contrary that $x \not D$. Then, there are feasible distinct plans $x^{\prime}$ and $x^{\prime \prime}$ such that $X=\frac{1}{2}\left(x^{\prime}+X^{\prime \prime}\right)$.

By hypothesis, there exists an item $j \varepsilon\{1,2, \ldots, M\}$ such that in a production period $b(1 \leq b \leq N)$, either $\left(\alpha_{j} / M_{\alpha}\right) L(b-1)=R_{1, b-1}(j)$ or $\left(\alpha_{j} / M_{\alpha}\right) L(b-1)=$ $R_{1, b-\beta_{j-1}}(j)$. Thereby, either $X^{\prime}$ or $X^{\prime \prime}$ is infeasible, or $X^{\prime}$ and $X^{\prime \prime}$ belong to different basic sets, which contradicts the assumption that both $X^{\prime}$ and $X^{\prime \prime}$ are feasible.

Thus the proof is completed. 
Now, it will be discussed about how to determine $x_{m+1(n)}^{*}$ required in each $a_{m n}$ computation for the extended problem. In fact, a similar procedure to that in section 2.3 can be constructed, since in view of Theorem 5 an optimal solution should form an at-least-one boundary sequence.

Theorem 6. For the extended problem, an optimal plan $x^{*}$ consists only of component $x_{m}^{* \prime} s(m=0,1, \ldots, N-1)$ satisfying the relation $x_{m+1(n)}^{*}=(L(n)-L(m))$ or $\left(L_{\beta}(n)-L(m)\right)$ for $0 \leq m<n \leq N$.

The results of Theorem 6 indicate that the solution procedure given in section 2.3 can be directly applied to the extended problem with the options for each $x_{m+1(n)}^{*}$ value. Therefore, it seems rather more difficult to search. a11 the associated extreme points.

\section{Multi-Facility Case with Backlog}

\section{1. $M$ products at each facility}

The model described in section 3 may be extended to the multi-facility problem treated in Zangwi11 [3], where $L$ individual facilities are linked together to form an acyclic network depicted in Fig.2.

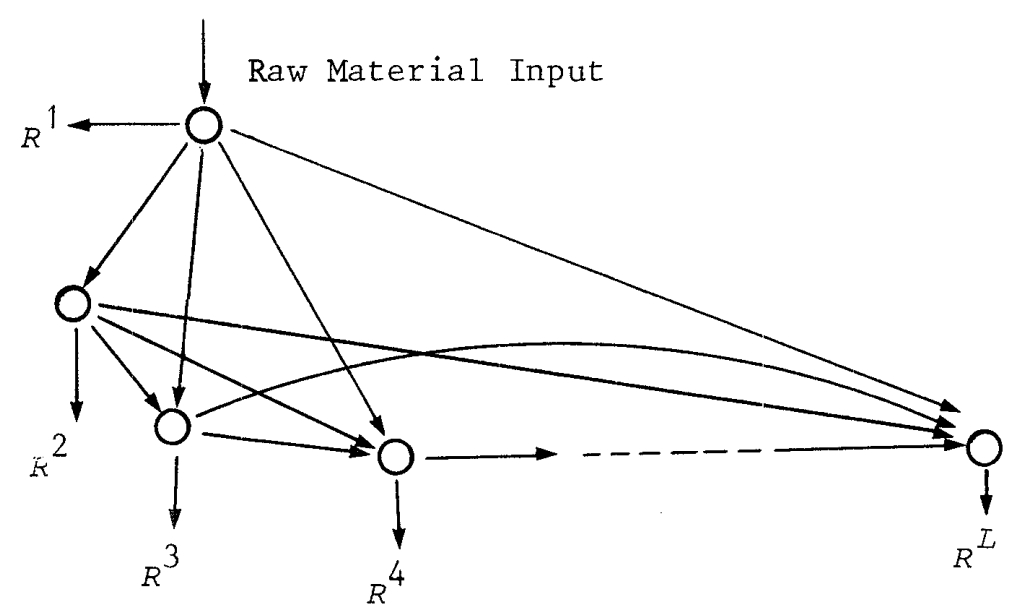

Fig. 2. The Acyc1ic Network with $L$ Facilities.

In the model, each facility except the first facility is allowed to receive inputs from raw materials or lower numbered facilities (not from itself or higher numbered facilities), then in each period manufacture $M$ specific products on its own production 1ine. Each product is then stored in inventory until needed either to satisfy demands for each product or to supply inputs to other faciliteies. 
$R^{b}=\left(r_{1}^{b}, r_{2}^{b}, \ldots, r_{N}^{b}\right), r_{i}^{b} \geq 0$, represents the market requirements vector for facility $b(b=1, \ldots, L)$ over the planning horizon $N$. Further, each $r_{t}^{b}$ itself is a demand vector composed of $M$ different demands, i.e., $r_{t}^{b}=\left\{r_{t j}^{b}\right\}$, where $r_{t j}^{b}$ represents the demand for item (product) $j(j=1,2, \ldots, M)$ at facility $b$ in period $t, 1 \leq t \leq N$. Assuming that all demands $r_{t j}^{b}$ are $f i x e d$ and known, we shall seek to determine the general form of the minimum cost production schedule (plan) that will specify how much each facility in the network should produce.

Let $x_{t}^{b} \geq 0$ be the production completed in period $t$ at facility $b$ and $I_{t j}^{b}$ the inventory of item $j$ at the end of period $t$ in facility $b$. Let $a_{j}^{b g} \geq 0$ be the number of units of facility $b^{\prime}$ s product $j$ required to produce one unit of facility $g^{\prime}$ s product $j$. It is assumed that facility $b$ can have a time lag $\lambda_{b}$ in production and thereby production started in period $t$ is completed in period $t+\lambda_{b}$. Then, the amount of item $j$ desired out of facility $b$ in period $t$ as inputs to other facilities is $\sum_{g=b+1}^{L} a_{j}^{b g} x_{t+\lambda_{g}}^{g}, j$, and the total demand on facility $b$ in period $t$ and the inventory level, denoted respectively by $y_{t j}^{b}$ and $I_{t j}^{b}$, are

$$
\begin{aligned}
& y_{t j}^{b}=r_{t j}^{b}+\sum_{g=b+1}^{L} a_{j}^{b g} x_{t+\lambda}^{g} \text {, and } \\
& I_{t j}^{b}=\sum_{h=1}^{t}\left(x_{h j}^{b}-y_{h j}^{b}\right) \text { for all } t, j \text { and } b .
\end{aligned}
$$

It is also assumed that each facility can backlog total demand for its product a certain fixed integral number of periods. Let $\beta_{j}^{b}$ represent the number of periods of backlog permitted for item $j$ at facility $b$. Then, the backlog limit for each item $j$ is $I_{t j}^{b} \geq-\sum_{h=t-\beta_{j}^{b}}^{b} y_{h j}^{b}$. Further, assume that $I_{N j}^{b}=0=I_{0 j}^{b}$ for all $b$ and $j$.

Let $z=\left(x^{1}, x^{2}, \ldots, x^{L}\right)=\left(x_{1}^{1}, \ldots, x_{N}^{1}, x_{1}^{2}, \ldots, x_{N}^{2}, \ldots, x_{1}^{I_{1}}, \ldots, x_{N}^{L}\right)$ denote the production schedule (vector) for the entire network, where $x^{b}=\left(x_{1}^{b}, \ldots, x_{N}^{b}\right)$ represents the production schedule for facility $b$ and each $x_{t}^{b}$ is decomposed into $M$ different products, $x_{t j}^{b}$, such that $x_{t}^{b} \cdot\left(\alpha_{j}^{b} / M_{\alpha}^{b}\right)=x_{t j}^{b}$ with $\alpha_{j}^{b}\left(\right.$ or $\left.M_{\alpha}^{b}\right)$ representing the fixed production ratio of item $j$ at facility $b$. Let $z^{b}=$ $\left(x^{b}, x^{b+1}, \ldots, x^{L}\right)$ be defined as a partial production schedule in facilities $b$ through $L$. Let $y^{b}=\left(y_{1}^{b}, y_{2}^{b}, \ldots, y_{N}^{b}\right)$ represent the total demand for facility $b$. $z^{b+1}$ completely specifies $Y^{b}$, so that it is convenient to write $y^{b}\left(z^{b+1}\right)$ to explicitly denote $Y^{b}$ s dependence on $z^{b+1}$. Then, a partial production vector 
$z^{g}$ is said to be feasible if the above constraints hold for all $b \geq g$ and all $t$. Similarly, if $z^{g+1}$ is feasible, $x^{g}$ is said to feasibly supply $z^{g+1}$ if $z^{g}=$ $\left(x^{g}, z^{g+1}\right)$ is feasible.

Although each facility in the network produces $M$ different items in each period, all the results obtained for the single-facility case hold, since the arguments at each facility are the same as for the problem in section 3 . We shall not repeat them all here in detail. However, Lemma 1 shall be proved to make the confirmation of the results easy.

Let $D$ be the dominant set, which is the union of the extreme points of all the $s^{L M N}$ basic sets, and $D^{b}$ denote the partial dominant set constructed from facilities $b$ through $L$.

Lemma 1. Let $z^{b+1}, \bar{z}^{b+1}, \tilde{z}^{b+1}$ be feasible partial. production vectors such that $\bar{z}^{b+1} \neq z^{b+1}$, and $z^{b+1}=\frac{1}{2}\left(\bar{z}^{b+1}+z^{b+1}\right), b \geq 1$. Assume $x^{b}$ feasibly supplies $z^{b+1}$ and that it forms an at-least-one boundary sequence for $Y^{b}\left(z^{b+1}\right)$. Then, there exist production vectors $\bar{x}^{b}$ and $\tilde{x}^{b}$ that feasibly supply $\bar{z}^{b+1}$ and $\tilde{z}^{b+1}$, respectively, such that $\left(x^{b}, z^{b+1}\right)=\frac{1}{2}\left[\left(\bar{X}^{b}, \bar{z}^{b+1}\right)+\left(\tilde{X}^{b}, \tilde{z}^{b+1}\right)\right]$. Furthermore, $I_{t}^{b} \geq 0$ iff $\bar{I}_{t}^{b} \geq 0$, and $I_{t}^{b} \geq 0$, iff $\tilde{I}_{t}^{b} \geq 0$ for all $t, t=1, \ldots, N$.

Proof: From the inventory constraints and the relation, $z^{b+1}(j)=$ $\frac{1}{2}\left(\tilde{z}^{b+1}(j)+\bar{z}^{b+1}(j)\right)\left(z^{b+1}(j)\right.$ denoting the partial production vector for item $j)$, there exist real values, $\delta_{t j}$, such that

$$
\delta_{t j}=\sum_{g=b+1}^{L} a_{j}^{b g_{\bar{x}}^{g}}+\sum_{g}, j-\sum_{g=b+1}^{L} a_{j}^{b g_{x} g} x_{t+\lambda}, j \text { and }\left|\delta_{t j}\right| \leq y_{t j}^{b} .
$$

Since $x^{b}$ forms an at-least-one boundary sequence for $y^{b}\left(z^{b+1}\right)$, there are integers $0=s(0, j) \leq s(1, j) \leq \ldots \leq s(N, j)=N$ for each item $j \varepsilon\{1,2, \ldots, M\}$ such that

$$
x_{t j}^{b}=\sum_{h=s}^{s(t, j)} y_{h j}^{b} .
$$

Define, for each item $j$,

$$
\begin{aligned}
& \tilde{x}_{t j}^{b}=\sum_{h=s(t-1, j)+1}^{s(t, j)}\left(y_{h j}^{b}-\delta_{h j}\right) \text { and } \\
& \tilde{x}_{t j}^{b}=\sum_{h=s}^{s(t, j)},(t-1, j)+1 \\
& \left(y_{h j}^{b}-\delta_{h j}\right) .
\end{aligned}
$$

Then, the proof can be completed just by following the proof steps of Lemma 1 in Zangwil1 [3].

By using the results of Lemma 1 and following the proof arguments of 
Theorem 1 in Zangwill [3], Theorem 7 (which characterizes the structure of the optimal solution) can then be easily proved.

Theorem 7. A feasible plan $z=\left(x^{1}, x^{2}, \ldots, x^{L}\right)$ consists only of each facility's at-least-one boundary sequence for each $z^{b+1}$ in $D^{b+1}$ for all b iff it is in $D$.

\subsection{Higher-numbered products at each facility}

Consider again the acyclic network problem with each facility allowed only to manufacture the higher-numbered but different items in each production period. That is, in each production period, facility $L$ produces a single item, facility $L^{-1}$ manufactures two different items (one for its own market requirements and the other one for inputs to facility $L$ ), and so on. In fact, $L=M$. Facility 1 is then required to manufacture $M$ different items in each production period to satisfy its own demands and supply inputs to other facilities.

Al1 of the results obtained for the problem in section 4.1 still hold. However, in comparison with that in section 4.1, this problem is rather simpler in determining an optimal production vector, since it has, in general, the much smaller number of basic sets, $2^{N M(M+1) / 2}$, and each of the extreme points at each facility can be directly searched by applying Theorem 5 in section 3 with each adjusted number of items.

\section{Conclusion}

In this paper, we have found a useful description of the structure of optimal plans which form at-least-one exact (boundary) requirement sequences. The optimal plan structures have also been shown to hold for the nonbacklog models on both single-facility and multi-facility systems. This solution characterization has then been incorporated in constructing a dynamic programming algorithm. The algorithm may be practically and efficiently used to find optimal solutions for smal1-sized problems.

It is further noticed that as an immediate extension, a capacitated multiproduct model can also be handled in the framework of this paper.

\section{Acknowledgement}

The author wishes to thank the referees for their numerous valuable comments and suggestions. 
[1] Florian, M. and Klein, M.: Deterministic Production Planning with Concave Costs and Capacity Constraints. Management Science, 18 (1971), 12-20.

[2] Wagner, H. A. and Whitin, T. M.: Dynamic Version of the Economic Lot Size Model. Management science, 5 (1959), 89-96.

[3] Zangwil1, W. I.: A Deterministic Multi-Product, Mu1ti-Facility Production and Inventory Mode1. Operations Research, 14 (1966), 486-507.

[4] Zangwil1, W. I.: A Backlogging Mode1 and a Multi-Echelon Model of a Dynamic Economic Lot Size Production System - A Network Approach. Manage-ment science, 15 (1969), 506-527.

C. S. SUNG: Industrial Engineering Department, Korea Advanced Institute of Science \& Technology, P. O. Box 150, Cheongryang Seoul, Korea 